\title{
The effect of a contralateral drone on the perceptual onset asynchrony of interaural tone sequences
}

\author{
SIMONE AKERBOOM and GERT TEN HOOPEN \\ University of Leiden, Leiden, The Netherlands
}

\begin{abstract}
Several studies reported that subjects have difficulty in processing a sequence of auditory signals if the sequence alternates between ears: Alternating speech and melodies are recognized poorly. If, however, the ears not receiving this information are "filled" with noise or with a lower constant-frequency tone (drone), recognition improves again. This improvement is due to the fact that the signal is subjectively alternating to a much lesser extent. The present study was an attempt to measure the subjective ambient bandwidth within which the interaural signal moves when a contralateral drone is supplied. It turned out that the signal, although alternating between ears, appeared to arise from the middle of the head. The results were discussed in terms of auditory illusions.
\end{abstract}

It has been shown that subjects have more difficulty in processing a sequence of auditory signals if the sequence is alternated between the ears rather than being presented to just one ear. Cherry and Taylor (1954) demonstrated that a spoken message that was switched back and forth between the ears (duty cycle 0.5 ) becomes least intelligible at 3 alternations/sec, although all of the speech signal is physically present at either one ear or the other. They interpreted these results as being a consequence of a limitation in the rate at which listeners can switch their attention.

Interestingly, Schubert and Parker (1956) found a strong increase in the intelligibility of alternating speech when the ear contralateral to the ear receiving the speech signal was stimulated with noise.

A related effect was described by Deutsch (1979). She found a marked decrement in the recognition of melodies when the components of the melody were switched between the ears. This decrement, however, could be largely nullified by adding a drone (a lower constant-frequency tone of $345 \mathrm{~Hz}$ ) to the ear opposite to the one receiving the melody. Deutsch mentioned that, in the latter condition, her subjects mislocalized the signals: "some perceived the melody on one side and the drone as localized on the other; others perceived the drone as in the center of the head and the melody as slowly shifting its position" (Deutsch, 1979, p. 403).

This study has been supported by a grant from the Netherlands Organization for the Advancement of Pure Research (ZWO). Requests for reprints should be sent to Gert ten Hoopen, Unit of Experimental Psychology, Hooigracht 15, 2312 KM Leiden, The Netherlands.
Deutsch referred to a related mislocalization effect found by Warren and Bashford (1976), who presented signals to one ear and noise to the other ear and reversed this situation every $500 \mathrm{msec}$. The signal appeared to be diffusely localized about the median sagittal plane, whereas the noise appeared to alternate between the ears.

Although the three studies mentioned above used different signals (Deutsch, 1979-melodies; Schubert \& Parker, 1956-speech; Warren \& Bashford, 1976tones and filtered speech), the addition of a contralateral drone or noise resulted in the same experience with regard to the perceived ambient excursions: the sequence of signals wandered from left to right and vice versa to a much lesser extent than when presented without contralateral stimulation. This mislocalization effect, that is, the narrowing of the subjective ambient bandwidth within which the signals move, obviously is the reason that the alternating speech and melodies are processed almost as efficiently as in the nonalternating condition.

It is not the purpose of the present study to explain this effect. Possible accounts for it have been offered by Deutsch (1979), Judd (1979), and Warren and Bashford (1976). The present investigation is an attempt to quantify the amount of subjective angular separation between the alternating signals as a result of the presence of contralateral stimulation.

Recently, a paradigm suitable for solving this latter question has been developed. Ten Hoopen, Vos, and Dispa (1982) presented monaural and interaural sequences of short tones to their subjects. The sequences comprised an unpredictable number of tones (6-17). In addition, the stimulus onset asynchrony (SOA) between the tones was varied $(125,167$, and $250 \mathrm{msec})$. The listener's task consisted of respond- 
ing as fast as possible to the end of the tone sequence. It turned out that the mean stop reaction time (stop RT) with interaural sequences was $24 \mathrm{msec}$ longer than with monaural sequences and was invariant with the value of the SOA. In a subsequent study (Akerboom, ten Hoopen, van der Schaaf, \& Olierook, Note 1), exactly the same invariant difference of 24 msec mean stop RT between interaural and monaural conditions was found with a much larger range of SOAs, namely, 40-2,130 msec.

These results were interpreted as follows: although the spacing of the tones in objective time is the same for both conditions (interaural and monaural), their subjective spacing in time is not. We coined the term "perceptual onset asynchrony" (POA) to stand for the subjective time between two successive tone percepts. We suggested that the stop-RT task requires that the listener continuously inspect his/her auditory memory for the duration of POA (not SOA) whether another tone arrives or not. When no subsequent tone arrives, the response is initiated. Response-initiation time was assumed to be the same for the monaural and interaural conditions. Thus, the 24-msec difference between interaural and monaural stop RTs reflects the difference between interaural and monaural POA.

This latter result is a quantification of the observation, first reported by Axelrod, Guzy, and Diamond (1968), that the apparent repetition rate of interaural sequences is slower than that of monaural sequences. A further study undertaken by Axelrod and Powazek (1972) revealed that the difference in apparent rate between alternating and nonalternating sequences became smaller with decreasing angular separation between the alternating tones. This finding was supported by a recent experiment at our laboratory (Akerboom et al., Note 1). Twenty-seven subjects performed the stop-RT task with location-alternating sequences of short noise bursts $(20 \mathrm{msec})$. The angular separation between the two noise-burst-emitting loudspeakers was varied between $0,30,60,90,120$, 150 , and $180 \mathrm{deg}$. In addition, the SOA of the sequences was varied among $100,200,300$, and $400 \mathrm{msec}$. It turned out that there was a significant main effect of angular separation on stop RT $[F(6,156)$ $=6.57, p<.001]$. The stop RT increased linearly with increasing angle between locations. The results are displayed in Table 1.

It should be evident from Table 1 that the POA difference between alternating and nonalternating sound sequences increases in a linear fashion with increasing angular separation. The logic underlying the present study is that a certain POA difference can be "translated" into the corresponding subjective angular separation by means of the linear relation between both variables. Thus, when the addition of contralateral drones to an interaural sequence results in a smaller subjective alternation of the signal, the POA difference should become smaller. The extent to
Table 1

Mean Stop RTs Dependent on Angular Separation (in Degrees) and Stimulus Onset Asynchrony (in Milliseconds)

\begin{tabular}{cccccc}
\hline \multirow{2}{*}{$\begin{array}{c}\text { Angular } \\
\text { Separation }\end{array}$} & 100 & 200 & 300 & 400 & Mean \\
\cline { 2 - 6 } & \multicolumn{5}{c}{ Stimulus Onset Asynchrony } \\
\hline 0 & 300 & 418 & 520 & 622 & 465 \\
30 & 305 & 422 & 518 & 627 & 468 \\
60 & 307 & 426 & 530 & 631 & 473 \\
90 & 309 & 424 & 529 & 630 & 473 \\
120 & 310 & 424 & 532 & 632 & 475 \\
150 & 315 & 433 & 530 & 634 & 478 \\
180 & 312 & 432 & 529 & 635 & 477 \\
\hline
\end{tabular}

Note-The linear regression line through the mean stop RTs has the equation: $R T=467+.07 \times$ angular separation in milliseconds $\left(r^{2}=.90\right)$.

which this happens reveals the amount of subjective alternation.

\section{METHOD}

\section{Subjects}

Twenty female and male students from the University of Leiden participated in the experiment and were paid for their services. Left- and right-ear thresholds were screened, the rejection criterion being a loss of more than $15 \mathrm{~dB}$ or a difference between left and right ear of more than $10 \mathrm{~dB}$. No subject had to be rejected.

\section{Stimulus Material and Design}

The stimulus material was constructed such that it approximated the conditions utilized by Deutsch (1979). Interaural, monaural, and dichotic sequences were generated by means of Coulbourn Instruments modules under the control of a microcomputer. The tones were pure sine-wave tones of either $550 \mathrm{~Hz}$ (tone) or $350 \mathrm{~Hz}$ (drone), with durations of $30 \mathrm{msec}$ and rise and fall times of $4 \mathrm{msec}$ each.

The monaural sequences comprised either the $550-\mathrm{Hz}$ tones or the 350-Hz drones. The interaural sequences also comprised either the $550-\mathrm{Hz}$ tones or the $350-\mathrm{Hz}$ drones. The dichotic sequences were constructed by adding the interaural drone sequence and the interaural tone sequence in a contralateral way. Thus, when one ear received the $550-\mathrm{Hz}$ tone, the other ear received the $350-\mathrm{Hz}$ drone, or vice versa.

In order to equalize the intensity of the three different presentation modes (interaural, monaural, dichotic), the 350- and $550-\mathrm{Hz}$ tones of the dichotic condition were each attenuated by $1.5 \mathrm{~dB}$. The SOA between the tones was 150, 250, 350, and $450 \mathrm{msec}$. The number of tones in a sequence could be $10,11,12$, $13,14,15,16$, or 17 .

Thus, there were 5 (interaural-550 or $350 \mathrm{~Hz}$; monaural-550 or $350 \mathrm{~Hz}$; dichotic) $\times 4$ (SOAs) $\times 8$ (number of tones) $=160$ different sequences that were blocked with respect to presentation mode and SOA. In each of the 20 blocks, the 8 sequences were randomized. The sequences within blocks were separated by a silent response interval of $2.5 \mathrm{sec}$.

\section{Procedure and Apparatus}

The subjects were tested individually in a sound-attenuated booth (Siemens). First, they were screened by means of a pure-tone audiometer (Peekel D66) and trained for the experiment; then they were presented with the 20 blocks, which were balanced across the subjects. The subjects were instructed to respond as soon as they were sure that the tone sequence was over by depressing a push button under the dominant index finger. The stop RTs, that is, the elapsed time between the end of the last tone and the depressing of the button, were registered by the computer. All apparatus except 
for the headphones (AKG-Cardan K140) and the push button were located outside the booth.

\section{RESULTS AND DISCUSSION}

The mean stop RTs for the monaural tone sequences and monaural drone sequences did not differ (both $524 \mathrm{msec}$ ). There was a slight difference of $13 \mathrm{msec}$ in mean stop RT for the interaural tone sequences $(538 \mathrm{msec})$ and the interaural drone sequences $(551 \mathrm{msec})$. This difference turned out to be significant $[F(1,19)=7.85, p<.02]$, which was a rather unexpected fact because, in a pilot study with 13 subjects, we did not find a significant difference in stop RT between interaural sequences with $350-\mathrm{Hz}$ drones and interaural sequences with $550-\mathrm{Hz}$ tones $[F(1,12)=1.88, p>.19]$. Hence, for further analysis, we decided to average the monaural stop RTs over both frequency conditions and also to average the interaural stop RTs over both frequency conditions. The mean stop RTs dependent on presentation mode and SOA are presented in Table 2.

We performed a within-subjects analysis of variance with the following factors: 3 presentation modes (monaural, interaural, dichotic), 4 SOAs $(150,250,350,450 \mathrm{msec}), 8$ numbers of tones $(10, \ldots, 17)$, and 20 subjects, all factors fixed except subjects. All factors yielded significant main effects. The main effect of SOA is an artifact of the stop-RT paradigm: longer SOAs imply longer POAs and hence longer stop RTs. The main effect of number of tones in the sequence is not relevant for the present question and is treated elsewhere (ten Hoopen et al., 1982).

The main effect of presentation mode (monaural, interaural, dichotic) was significant $[F(2,38)=15.90$, $\mathrm{p}<.0001$ ). As Table 2 shows, this effect can be ascribed totally to the difference between the interaural mode on the one hand $(544 \mathrm{msec})$ and the monaural and dichotic modes on the other hand (both $524 \mathrm{msec}$ ). The difference of $20 \mathrm{msec}$ corresponds quite closely to the value established earlier at our laboratory $(24 \mathrm{msec})$. The mean stop RT for the dichotic sequences (tone and drone) is precisely the same as that for the monaural sequences. However, this does not hold at all SOAs. At the SOAs of 250 and $450 \mathrm{msec}$, both stop RTs are the same, but at

Table 2

Mean Stop Reaction Times

\begin{tabular}{lccccc}
\hline & \multicolumn{5}{c}{$\begin{array}{c}\text { Stimulus Onset Asynchrony } \\
\text { Between Tones }\end{array}$} \\
\cline { 2 - 6 } $\begin{array}{c}\text { Presentation } \\
\text { Mode }\end{array}$ & 150 & 250 & 350 & 450 & Mean \\
\hline Monaural & 352 & 468 & 584 & 694 & 524 \\
Interaural & 376 & 490 & 598 & 714 & 544 \\
Dichotic & 363 & 468 & 574 & 691 & 524 \\
\hline
\end{tabular}

Note-All values in milliseconds.

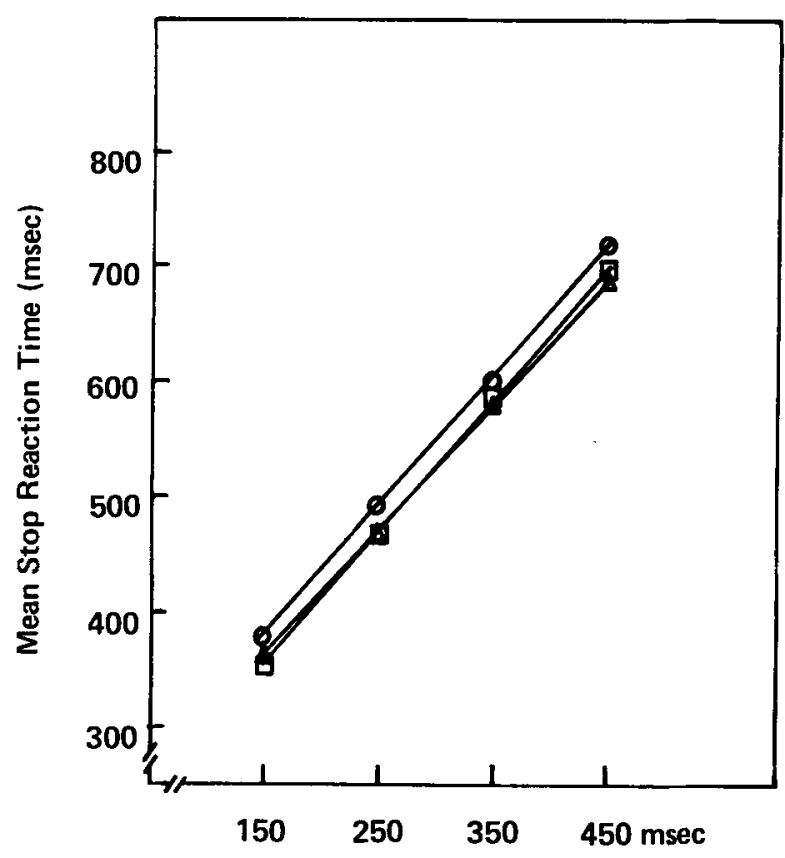

Stimulus Onset Asynchrony

Figure 1. Mean stop reaction times as a function of presentation mode (interaural, monaural, or dichotic) and stimulus onset asynchrony. For each of the presentation modes, the best-fiting linear regression line is inserted. The equations are: $\tilde{\mathbf{R T}} \tilde{T}_{\text {lat }}=208+1.12$ SOA msec $(0-O)$, $\widetilde{R T}_{\operatorname{mon}}=182+1.14$ SOA msec $(\square-\square)$, $\tilde{R} T_{d k h}=197+1.0950 A$ msec $(\Delta-\Delta)$. All $\mathrm{r}^{2}$ s exceed 0.98 .

SOAs of 150 and $350 \mathrm{msec}$, the dichotic and monaural stop RTs differ by about $10 \mathrm{msec}$, although in a different direction. The interaction between presentation mode and SOA, however, was not significant ( $p>$.23). Thus, these departures appear not to be systematic.

We therefore plotted the mean stop RTs dependent on presentation mode and SOA in Figure 1 and fitted three linear regression lines, one for each presentation mode. As one can see, the slopes of these three lines are almost equal. The dichotic and monaural regression lines cover each other, whereas the interaural equation is elevated by about $20 \mathrm{msec}$.

Recall from the introduction that one could estimate, by means of Table 1 , the perceived angular separation by converting the POA difference between the dichotic and monaural conditions into the corresponding angle. It must be evident that this angle amounts to $0 \mathrm{deg}$, because the POA difference is $0 \mathrm{msec}$. This implies that the ambient excursions of the sounds (objectively $180 \mathrm{deg}$ ) are reduced by adding the drones to a subjective extent corresponding to 0 deg. This finding is corroborated by the introspections of our subjects. They reported that the perceived sounds alternated between a high and a low pitch but that they all appeared somewhere in the 
middle of the head, each sound arising only a tiny little bit aside from its predecessor.

These introspective reports, however, might also be regarded as a criticism of our choice of the stimulus material for the present investigation. We prepared our stimuli in order to mimic the Deutsch (1979), the Schubert and Parker (1956), and the Warren and Bashford (1976) conditions. All these conditions yielded two streams: melodies and drones, speech and noise, and signals and noise, respectively. In addition, in all three cases, the two streams were also discriminable with regard to their ambient behavior. For instance, one stream remained positioned about the medial sagittal plane, whereas the other shifted back and forth between the ears. In the dichotic condition of the present study, only one stream was perceived. Nevertheless, our POA technique firmly established that this stream did not move through phenomenal auditory space.

It appears to us that the present dichotic stimulation yielded perceptual results that are very much related to the octave illusion (Deutsch, 1975, 1980). Deutsch presented sequences comprising dichotic pairs of 400 $800-\mathrm{Hz}$ tones, the tones reversing between the ears every $250 \mathrm{msec}$. Her subjects stated that they heard only one stream of sounds, alternating between a high and a low pitch and at the same time alternating between the ears. Our stream also alternated between a high and a low pitch, and, although a locationalternating effect was barely detectable, careful listening revealed that the high pitch arose slightly to the right of the median sagittal plane and the low pitch slightly to the left thereof.

Thus it seems to us that the "frequency part" of the octave illusion, that is, the suppression of the frequency presented to the left ear by the frequency presented to the right ear, also holds for our dichotic pairs of tones-350-550 $\mathrm{Hz}$-even though this ratio is far from an octave. On the other hand, the "location part" of the octave illusion hardly shows up with our material. Hence, it seems to be necessary that the dichotic tones bear an octave relation in order to invoke the illusory alternation between ears, although a slight departure of $10-15 \mathrm{~Hz}$ does not harm the illu- sion (Deutsch \& Roll, 1976). The question remains, however, whether the illusory location alternation, yielded as part of the "perfect" octave illusion, corresponds to an objective alternation of $180 \mathrm{deg}$ angular separation or not. Our POA technique might provide the answer.

\section{REFERENCE NOTE}

1. Akerboom, S. P., ten Hoopen, G., van der Schaaf, T. W., \& Olierook, P. Auditory spatial alternation transforms auditory time. Manuscript submitted for publication, 1983.

\section{REFERENCE}

Axelnod, S., Guzy, L. T., \& Diamond, I. T. Perceived rate of monotic and dichotically alternating clicks. Journal of the Acoustical Society of America, 1968, 43, 51-55.

Axelrod, S., \& Powaze K, M. Dependence of apparent rate of alternating clicks on azimuthal separation between sources. Psychonomic Science, 1972, 26, 217-218.

Cherry, E. C., \& TAYLOR, W. K. Some further experiments upon the recognition of speech, with one and with two ears. Journal of the Acoustical Society of America, 1954, 26, 554-559.

Deutsch, D. Musical illusions. Scientific American, 1975, 233, 92-104.

Deutsch, D. Binaural integration of melodic patterns. Perception \& Psychophysics, 1979, 25, 399-405.

Deutsch, D. The octave illusion and the what-where connection. In R. S. Nickerson (Ed.), Attention and performance VIII. Hillsdale, N.J: Erlbaum, 1980.

Deutscr, D., \& Roll, P. L. Separate "what" and "where" decision mechanisms in processing a dichotic tonal sequence. Journal of Experimental Psychology: Human Perception and Performance, 1976, 2, 23-29.

Judd, T. Comments on Deutsch's musical scale illusion. Perception \& Psychophysics, 1979, 26, 85-92.

Schubert, E. D., \& Parker, C. D. Addition to Cherry's findings on switching speech between the two ears. Journal of the Acoustical Society of America, 1956, 27, 792-794.

TEN Hoopen, G., Vos, J., \& DisPa, J. Interaural and monaural clicks and clocks: Tempo difference versus attention switching. Journal of Experimental Psychology: Human Perception and Performance, 1982, 8, 422-434.

Warren, R. M., \& Bashford, J. A. Auditory contralateral induction: An early stage in binaural processing. Perception \& Psychophysics, 1976, 20, 380-386.

(Manuscript received July 7, 1982;

revision accepted for publication February 2, 1983.) 